\title{
Maintaining cognitive function with internet use: a two-country, six-year longitudinal study
}

\author{
Jessica Berner, ${ }^{1,2}$ Hannie Comijs, ${ }^{2,3}$ Sölve Elmståhl, ${ }^{4}$ Anna-Karin Welmer, ${ }^{5}$ \\ Johan Sanmartin Berglund, ${ }^{6}$ Peter Anderberg, ${ }^{6}$ and Dorly Deeg ${ }^{1,2}$ \\ ${ }^{1}$ Department of Epidemiology and Biostatistics, VU University Medical Center, Amsterdam, the Netherlands \\ ${ }^{2}$ Amsterdam Public Health Research Institute, Amsterdam, the Netherlands \\ ${ }^{3}$ Department of Psychiatry, VU University Medical Centre, Amsterdam, the Netherlands \\ ${ }^{4}$ Lund University Sweden, Skåne University Hospital, Lund, Sweden \\ ${ }^{5}$ Aging Research Center, Karolinska Institutet, Stockholm, Sweden \\ ${ }^{6}$ Department of Health, Blekinge Institute of Technology, Karlskrona, Sweden
}

Objectives: Maintaining good cognitive function with aging may be aided by technology such as computers, tablets, and their applications. Little research so far has investigated whether internet use helps to maintain cognitive function over time.

Design: Two population-based studies with a longitudinal design from 2001/2003 (T1) to 2007/2010 (T2).

Setting: Sweden and the Netherlands.

Participants: Older adults aged 66 years and above from the Swedish National Study on Ageing and Care $(\mathrm{N}=2,564)$ and from the Longitudinal Aging Study Amsterdam $(\mathrm{N}=683)$.

Measurements: Internet use was self-reported. Using the scores from the Mini-Mental State Examination (MMSE) from T1 and T2, both a difference score and a significant change index was calculated. Linear and logistic regression analysis were performed with difference score and significant change index, respectively, as the dependent variable and internet use as the independent variable, and adjusted for sex, education, age, living situation, and functional limitations. Using a meta-analytic approach, summary coefficients were calculated across both studies.

Results: Internet use at baseline was $26.4 \%$ in Sweden and $13.3 \%$ in the Netherlands. Significant cognitive decline over six years amounted to $9.2 \%$ in Sweden and $17.0 \%$ in the Netherlands. Considering the difference score, the summary linear regression coefficient for internet use was -0.32 (95\% CI: $-0.62,-0.02)$. Considering the significant change index, the summary odds ratio for internet use was 0.54 (95\% CI: 0.37 , $0.78)$.

Conclusions: The results suggest that internet use might play a role in maintaining cognitive functioning. Further research into the specific activities that older adults are doing on the internet may shine light on this issue.

Key words: older adults, internet use, significant cognitive decline, longitudinal study, Sweden, the Netherlands

\section{Introduction}

Cognition represents a spectrum of higher order cerebral functions (Cohen et al., 2016), and it is mostly agreed that with age change occurs in memory, information processing and dividing attention. These changes tend to be associated with, yet not

Correspondence should be addressed to: Prof. dr. D.J.H. Deeg, Department of Epidemiology and Biostatistics, VU University Medical Center, P.O. Box 7057, 1007 MB Amsterdam, the Netherlands. Telephone: +3120 444 6767. Email: djh.deeg@vumc.nl. Received 11 Nov 2018; revision requested 26 Jan 2019; revised version received 15 May 2019; accepted 15 May 2019. determined by, chronological age. There is enormous variability in the rate of cognitive decline in older adults across cohorts and individuals (Baltes, 1997).

It is difficult to pinpoint what the reasons are for changes in cognitive functioning. As age increases, the brain undergoes a series of changes, namely in gray and white matter, neurochemical alterations, blood flow reductions, and synaptic degeneration. Some have claimed that with age there is a decrease in the speed of processing information in the cognitive system (Salthouse, 1996). Problems 
can arise, therefore, in learning and in retrieving information, resulting in memory problems (Jolles et al., 1995).

Research has indicated that it is possible to compensate for changes in the aging brain, as seen with some older adults (Cabeza et al., 2002). Using the internet may help older people to train certain tasks, which in turn may help them to avoid losses and foster maintenance. For example, online shopping may help older people to stay independent, and playing games on the internet may stimulate information processing speed.

Promoting healthy cognitive aging is key in supporting our aging societies. The proportion of older adults is increasing in our communities, so it benefits the whole society to help the elderly sustain a healthy aging mind as the contrary leads to high social, personal, and healthcare costs.

Today's information society offers a wide range of services, including information which may facilitate healthy aging for older adults (Sanchez-Valle et al., 2017). Internet usage is rising among older adults, with a major increase in usage in just the last few years. In Europe, the percentage of people 65-74 years of age who had used the internet in the last three months was $27 \%$ in 2010 compared to $49 \%$ in 2016 (Eurostat, 2017).

Little research has been done so far to investigate whether internet use is a means to maintain cognitive function over time. One British study investigated whether internet use reduced cognitive decline indicating that in addition to higher education and no functional impairments, internet or email use were specifically associated with better performance in delayed recall (Xavier et al., 2014). Other research has indicated that internet use by older adults may promote well-being and may impact independence and health, which in turn can lead to a positive effect on cognitive function (Ashby et al., 1999). A review of interventions on sustaining and testing cognitive performance indicated that many studies were weak on the fact that the samples and context (such as intensity of usage) were heterogeneous, making generalizations difficult (Reijnders et al., 2013).

The current study adds to the scarce available evidence by addressing the question whether internet use is associated with change in cognitive functioning in two longitudinal studies from Sweden and the Netherlands, two countries with similar welfare levels and some of the highest percentages of older adult internet users. One difference between these two countries, which may be relevant to internet use, is the much lower population density in Sweden than in the Netherlands (Berner et al., 2015). We hypothesize that there is less decline in cognitive function in older adults who use the internet.

\section{Methods}

\section{Study samples}

In order to achieve a stronger evidence base, data are used from two population-based longitudinal studies on aging with similar designs.

The Swedish data is from the Swedish National Study on Aging and Care (SNAC), which has been an ongoing study since 2001 with the aim to investigate, monitor, and evaluate the health of the aging population of Sweden. There are four participating regions: Kungsholmen (Stockholm), Skåne, Nordanstig, and Blekinge. Several questionnaires and interviews were used. Participants were randomly selected from 11 age cohorts of people 60 years and older. From the eligible persons, $66.4 \%$ participated in the baseline (2001/2003) examination, with six-year follow-ups. For a more detailed description of SNAC, see Lagergren et al. (2004).

The Dutch data is from the Longitudinal Aging Study Amsterdam (LASA), which investigates predictors and consequences of changes in social, mental, and physical functioning in the aging population of the Netherlands. Random samples of older people were drawn from population registers in 11 municipalities in three regions in the Netherlands. The first cohort (aged 55-85) was interviewed in 1992/1993 with a response rate of $(62 \%)$, and since, they were followed-up with every three years. For a more detailed description of LASA, see Huisman et al. (2011). For the current study, the LASA measurement cycle $(2001 / 2002)$ closest in time to the SNAC baseline (2001/2003) was considered as the baseline.

The authors selected from the baseline of SNAC and the LASA cycle a sample, which consisted of a similar age group, who had answered the question on internet use and who had taken the Mini-Mental State Examination (MMSE) at two time points T1 (2001-2003) and T2 (2007-2010).

The sample size in SNAC at T1 of people 66 years of age and above was $\mathrm{N}=5,628$. We selected those who answered the question on internet use, reducing the sample size to $\mathrm{N}=4,840$. We then selected those who answered the MMSE at T1, reducing the sample to $\mathrm{N}=4,693$, and those who answered the MMSE at T2, reducing the sample to $\mathrm{N}=2,872$. This gave us a Swedish sample of size $\mathrm{N}=2,872$. There were $14.0 \%$ of older Swedish adults who did not answer the question on internet use at T1. They were older (mean age 77), had more functional limitations and lived alone more often.

At first, the sample size in LASA was $\mathrm{N}=1,412$. After selecting those 66 years and older, the sample was reduced to $N=1,351$. Then selecting those who answered the question of internet use reduced the sample to $N=1,236$. Selecting the participants who 
answered the MMSE at $\mathrm{T} 1(\mathrm{~N}=1,230)$ and then at $\mathrm{T} 2$ reduced the sample to $\mathrm{N}=683$. The final Dutch sample used in this study was $\mathrm{N}=683$. There were $8.5 \%$ of respondents who did not answer the question on internet use. These people were older (mean age 82), living alone, and had more functional limitations.

\section{Measures}

From the measures deemed relevant, we selected those that the two studies had in common.

The question about internet use was asked in a way to garner a yes or no response from the subject. Cognitive functioning was measured using the MMSE, a screening test of cognitive impairment in older persons (Folstein et al., 1975). This test consists of 23 items covering the domains of orientation, memory, attention, language and visual construction. The scores range from 0 to 30, with higher scores reflecting better cognitive functioning. Respondents with an MMSE score under 11 were excluded. It was deemed that people scoring 11 and lower would not be able to properly answer the question about internet use. The MMSE was used as a continuous measure in this study.

As potential confounders of the association between internet use and cognitive function, the following were selected: education, gender, age, functional limitations, and living alone or not. With respect to education, the last century has seen an expansion in education where more people attend school for a longer period of time, which is associated with an increase in cognitive scores over time. Education levels were dichotomized into lower and higher education (secondary school and above).

Functional limitations have been reported to predict cognitive decline (Ellwardt et al., 2015) and also to hamper computer use (Berner et al., 2016). Functional limitations were assessed with a self-reported questionnaire based on a selection of questions from the Instrumental Activities of Daily Living questionnaire (Lawton and Brody, 1969) and the Organisation for Economic Co-operation and Development (OECD) scale (McWhinnie, 1981). The questions asked about walking up and down 15 flights of steps, cutting one's toenails, being able to use public transport, walking for five minutes without stopping, being able to sit and rise from a chair, and being able to dress/ undress oneself. A functional limitation was defined as having difficulty or not being able to do the activity and scored as 1 ; having no difficulty was scored as 0 . The six items were summed, so that the higher the sum score the more problems in daily functioning. It was used as a continuous variable in the analyses.

The living situation (i.e. living alone or not) might be a potential confounder because living with someone is assumed to lead to more social behavior such as talking and engaging with others. Studies have indicated that living alone can lead to a two-times greater likelihood of cognitive decline compared to not living alone (Van Gelder, et al., 2006). Moreover, living with someone has been shown to be associated with more internet use, as observed in a study conducted on older adults living in rural and urban Sweden (Berner et al., 2015). Living with the spouse, with family, or in an institution/home was considered as not living alone and was coded as 0 , while living alone was coded as 1 .

\section{Analyses}

The Swedish sample was weighted to the Dutch sample by gender and age in five-year strata (66-69.9; 70-74.9; 75-79.9; 80-84.9; and 85+). This was done to have comparable age and gender distributions for Sweden and the Netherlands.

The first approach to capture the change in cognitive function between the waves T1 (2001/ 2003) and T2 (2007/2010) was to calculate the difference in the MMSE score, i.e. the score at $\mathrm{T} 1$ - the score at $\mathrm{T} 2$.

A difference score is not an optimal approach because of the likelihood of regression to the mean. Moreover, a difference of, for example, two points may mean a lot in highly functioning individuals but may mean less in individuals with poor functioning. Therefore, in addition, a significant decline in cognitive scores was used. The Edwards-Nunnally (EN) index determines significant change in the cognitive score between two measurements, thereby avoiding regression to the mean (Speer and Greenbaum, 1995). To determine significant cognitive decline (yes/no), the following formula was used: $\mathrm{X}_{\mathrm{T} 2}<$ (Cronbach's $\alpha^{\star}\left(\mathrm{X}_{\mathrm{T} 1}-\right.$ mean $)+$ mean $-1.960^{\star}$ Standard error). $\mathrm{X}_{\mathrm{T} 1}$ and $\mathrm{X}_{\mathrm{T} 2}$ refer to the individual's raw score on $\mathrm{T} 1$ and $\mathrm{T} 2$, respectively.

To illustrate how the scores are distributed in the two categories, no significant decline and significant decline, the difference score of $\mathrm{T} 1-\mathrm{T} 2$ was cross tabulated with the significant cognitive decline score computed with the EN index. A significant decline represented a difference of at least four points on the MMSE (Supplementary material).

Two analytic approaches were used for both the Swedish and Dutch studies. First, in linear regression analysis, the MMSE difference score was the dependent variable. Secondly, logistic regression was used with the EN index as the dependent variable. In both analyses, the first model was adjusted only for cognitive score at baseline in order to rule out reverse causation, i.e. those initially more cognitively able may be using the internet more 
Table 1. Distribution of the baseline characteristics. The Swedish data is weighted to the Dutch data by gender and age

\begin{tabular}{|c|c|c|c|c|}
\hline & \multicolumn{2}{|c|}{ SWEDEN } & \multicolumn{2}{|c|}{ The Netherlands } \\
\hline & T1 (2001/2003) & T2 (2007/2010) & T1 $(2001 / 2002)$ & T2 (2008/2009) \\
\hline $\mathrm{N}$ & 2872 & 2872 & 683 & 683 \\
\hline Age range & $66-96$ & $72-96$ & $66-94$ & $72-100$ \\
\hline Age mean & 73.7 & 79.6 & 74.0 & 81.1 \\
\hline Female & $1682(58.6 \%)$ & $1682(58.6 \%)$ & $401(58.7 \%)$ & $401(58.7 \%)$ \\
\hline Male & $1190(41.4 \%)$ & $1190(41.4 \%)$ & $282(41.3 \%)$ & $282(41.3 \%)$ \\
\hline Education lower & $1363(47.6 \%)$ & $1363(47.6 \%)$ & $560(82.0 \%)$ & $560(82.0 \%)$ \\
\hline Education higher & $1500(52.4 \%)$ & $1500(52.4 \%)$ & $123(18.0 \%)$ & $123(18.0 \%)$ \\
\hline Living alone & $1209(42.2 \%)$ & $1347(62.5 \%)$ & $263(38.6 \%)$ & $350(51.3 \%)$ \\
\hline Not living alone & $1657(57.8 \%)$ & $810(37.5 \%)$ & $419(61.4 \%)$ & $332(48.7 \%)$ \\
\hline No functional limitations & $1906(77.2 \%)$ & $1521(61.1 \%)$ & $564(83.1 \%)$ & $381(58.1 \%)$ \\
\hline At least one functional limitation & $563(22.8 \%)$ & $966(38.9 \%)$ & $115(16.9 \%)$ & $275(41.9 \%)$ \\
\hline Internet use & $758(26.4 \%)$ & $843(31.3 \%)$ & $91(13.3 \%)$ & $173(27.2 \%)$ \\
\hline No internet use & $2114(73.6 \%)$ & $1848(68.7 \%)$ & $592(86.7 \%)$ & $462(72.8 \%)$ \\
\hline
\end{tabular}

and may be declining less. The second model was additionally adjusted for the following independent variables: internet use, gender, age, education, functional limitations, and living situation. The results were synthesized using a meta-analytic approach in R-studio with inverse weights for study size. As there were only two studies, no heterogeneity could be determined.

\section{Results}

Table 1 describes the sample characteristics for Sweden and the Netherlands in time T1 (20012003) and T2 (2007-2010). After applying weights to the Swedish sample, in both samples the initial age was 74 years, and $59 \%$ was female. Both samples had comparable scores with respect to functional limitations, cognitive mean scores, and people living alone. However, for education, the percentages were clearly different; the Dutch sample being less educated than the sample from Sweden.

Among older adults in Sweden, internet use at baseline was $26.4 \%$, whereas in the Netherlands it was $13.3 \%$. Over time, internet use increased by $4.9 \%$ points in Sweden and $13.9 \%$ points in the Netherlands.

Table 2 presents the cognitive scores as well as the significant change in cognitive score (EN index). In the Netherlands, the mean MMSE score is 27.8 at $\mathrm{T} 1$ and in Sweden, 28.1. Both countries had a decrease in mean MMSE score at T2. The EN index showed that $9.2 \%$ of the participants in Sweden and $17.0 \%$ in the Netherlands had a cognitive decline.

By means of linear regression analysis, we studied whether internet use was associated with change in
Table 2. MMSE scores at T1 and T2 and significant cognitive decline between $\mathrm{T} 1$ and $\mathrm{T} 2$ according to the Edwards-Nunnally index

\begin{tabular}{|c|c|c|}
\hline MMSE SCORE & SwEDEN & The Netherlands \\
\hline Score $\mathrm{T} 1$ mean $(\mathrm{SD})$ & $28.1(1.99)$ & $27.8(1.98)$ \\
\hline Score $\mathrm{T} 2$ mean $(\mathrm{SD})$ & $27.2(2.60)$ & $26.7(3.10)$ \\
\hline \multicolumn{3}{|c|}{ Significant change between $T 1 \& \mathbf{T} 2$} \\
\hline Decline & $295(9.2 \%)$ & $116(17.0 \%)$ \\
\hline No decline & $2607(90.8 \%)$ & $567(83.0 \%)$ \\
\hline
\end{tabular}

cognitive scores (Table 3). A first model only including a baseline cognitive score showed that internet use was significantly associated with reduced cognitive decline over time in both cohorts with regression coefficients -0.70 in Sweden and -0.85 in the Netherlands. In both cohorts, this association became weaker when adjusting for other variables, but remained significant; using meta-analysis, the adjusted summary regression coefficient was -0.32 (95\% CI: - 0.62, - 0.02). In the Swedish cohort, the covariates of gender, age, living alone, and functional limitations were also significantly associated with a decrease in cognitive score. In the Dutch cohort, only age was additionally significant when associated with a decrease in cognitive score.

Regarding the association between internet use and significant decline as defined by the EN index Table 4 shows that in Sweden internet use was negatively associated with decrease in cognitive function (OR: $0.34,95 \% \mathrm{CI}: 0.23,0.51$ ). When adjusted for covariates, this association was attenuated but remained significant. In the Netherlands, internet use was not significantly associated with significant cognitive decline (OR: $0.56,95 \% \mathrm{CI}$ : $0.25,1.03)$. Adding covariates again attenuated 
Table 3. Linear regression analysis (unstandardized coefficients) of change in cognitive score (T1-T2), with internet use, gender, education, age, living alone, functional limitations, and baseline cognitive score

\begin{tabular}{|c|c|c|c|c|c|c|c|c|c|}
\hline \multirow[b]{2}{*}{ Model 1: } & \multicolumn{3}{|c|}{ SWEDEN } & \multicolumn{3}{|c|}{ The Netherlands } & \multicolumn{3}{|c|}{$\begin{array}{l}\text { META-ANALYSIS: SUMMARY } \\
\text { REGRESSION COEFFICIENT }\end{array}$} \\
\hline & B & $95 \% \mathrm{CI}$ & $\mathrm{P}$ value & B & $95 \% \mathrm{CI}$ & $\mathrm{P}$ value & $\mathrm{B}$ & $95 \% \mathrm{CI}$ & $\mathrm{P}$ value \\
\hline Internet use & -0.70 & $-0.92,-0.48$ & $<0.001$ & -0.85 & $-1.47,-0.23$ & 0.007 & -0.72 & $-0.92,-0.51$ & $<0.001$ \\
\hline MMSE at T1 & 0.25 & $0.20,0.30$ & $<0.001$ & 0.26 & $0.15,0.36$ & $<0.001$ & & & \\
\hline \multicolumn{10}{|l|}{ Model 2: } \\
\hline Internet use & -0.24 & $-0.47,-0.02$ & 0.035 & -0.63 & $-1.26,0.00$ & 0.050 & -0.32 & $-0.62,-0.02$ & 0.038 \\
\hline Gender ( $\mathrm{F}$ versus $\mathrm{M}$ ) & -0.37 & $-0.57,-0.18$ & $<0.001$ & -0.26 & $-0.71,0.20$ & 0.268 & & & \\
\hline Education & -0.27 & $-0.46,-0.07$ & 0.008 & -0.39 & $-0.94,0.17$ & 0.173 & & & \\
\hline Age & 0.10 & $0.08,0.11$ & $<0.001$ & 0.12 & $0.09,0.16$ & $<0.001$ & & & \\
\hline Living alone/not & -0.21 & $-0.40,-0.01$ & 0.042 & 0.37 & $-0.09,0.83$ & 0.116 & & & \\
\hline Functional limitations & 0.26 & $0.14,0.39$ & $<0.001$ & 0.10 & $-0.20,0.39$ & 0.520 & & & \\
\hline MMSE at $\mathrm{T} 1$ & 0.34 & $0.29,0.40$ & $<0.001$ & 0.32 & $0.22,0.43$ & $<0.001$ & & & \\
\hline
\end{tabular}

$\mathrm{B}=$ Unstandardized regression coefficient; $\mathrm{CI}=$ Confidence Interval.

Table 4. Logistic regression analysis (Odds Ratios) of significant decline in cognitive score, with internet use, gender, education, age, living alone, functional limitations, and baseline cognitive score

\begin{tabular}{|c|c|c|c|c|c|c|c|c|c|}
\hline \multirow[b]{2}{*}{ Model 1: } & \multicolumn{3}{|c|}{ SwEDEN } & \multicolumn{3}{|c|}{ The Netherlands } & \multicolumn{3}{|c|}{$\begin{array}{c}\text { META-ANALYSIS: } \\
\text { SUMMARY ODDS RATIO }\end{array}$} \\
\hline & OR & $95 \% \mathrm{CI}$ & $P$ value & OR & $95 \% \mathrm{CI}$ & $P$ value & OR & $95 \% \mathrm{CI}$ & $P$ value \\
\hline Internet use & 0.34 & $0.23,0.51$ & $<0.001$ & 0.56 & $0.25,1.03$ & 0.116 & 0.40 & $0.25,0.62$ & $<0.001$ \\
\hline MMSE at T1 & 0.84 & $0.79,0.88$ & $<0.001$ & 0.90 & $0.82,0.99$ & 0.025 & & & \\
\hline \multicolumn{10}{|l|}{ Model 2: } \\
\hline Internet use & 0.52 & $0.34,0.80$ & 0.003 & 0.61 & $0.28,1.31$ & 0.207 & 0.54 & $0.37,0.78$ & 0.001 \\
\hline Gender ( $F$ versus $M)$ & 0.84 & $0.63,1.11$ & 0.208 & 0.47 & $0.53,1.34$ & 0.474 & & & \\
\hline Education & 0.93 & $0.70,1.23$ & 0.611 & 1.06 & $0.59,1.92$ & 0.833 & & & \\
\hline Age & 1.09 & $1.07,1.12$ & $<0.001$ & 1.12 & $1.08,1.16$ & $<0.001$ & & & \\
\hline Living alone/not & 0.96 & $0.72,1.27$ & 0.765 & 1.42 & $0.87,2.32$ & 0.158 & & & \\
\hline Functional limitations & 1.26 & $1.11,1.43$ & $<0.001$ & 0.90 & $0.67,1.20$ & 0.466 & & & \\
\hline MMSE at T1 & 0.90 & $0.84,0.95$ & $<0.001$ & 0.92 & $0.83,1.03$ & 0.141 & & & \\
\hline
\end{tabular}

$\mathrm{OR}=$ Odds Ratio; $\mathrm{CI}=$ Confidence Interval.

these associations, but in Sweden the association remained significant. Meta-analysis showed a significant adjusted summary effect across the two studies (OR: 0.54, 95\%CI: 0.37/0.78). In Sweden, the covariates that showed significance were age and functional limitations; in the Netherlands, again, only age showed significance.

\section{Discussion}

This study sought to explore whether the cognitive activity of using the internet was associated with less cognitive decline over time in older adults 66 years of age and above. After adjustment for confounders in the linear model, internet use was significantly associated with the six-year difference in MMSE score in both countries. In the logistic regression model, with significant decline in cognitive score, in the Netherlands there was no significant association between internet use and cognitive decline after adjustment for covariates, whereas in Sweden, this association remained significant. Using a metaanalytic approach, the summary effect was significant, indicating that internet users have only half the chance of significant cognitive decline than noninternet users.

The linear regression analyses of the MMSE difference score showed that internet use is associated with a relatively small cognitive decline, as difference scores of one, two, or three points were most common (see Supplementary material). This small difference may have less relevance with regard to true cognitive decline. Significant decline using the EN index started only at a four points difference. Notably, internet use showed a consistent association even with this more severe cognitive decline.

The difference in strength of the findings in Sweden and the Netherlands might be explained 
by several factors. In Sweden, people started using the internet earlier than in the Netherlands. Daily use by Swedish older adults could therefore be more rooted than in the Netherlands. Also, the types of use may differ between Swedish and Dutch older adults. It may be that the tasks that are done online are key in the association of cognitive maintenance. Our study was not able to compare types of use; future studies are necessary to further examine this.

This study used population-based, longitudinal data from two countries, and it used the same design and regression models. This replication demonstrated for each country similar results in the main association, i.e. that internet use was associated with less subsequent cognitive decline. However, differences were found in the association of covariates. The fact that the relevant confounders differed between Sweden and the Netherlands suggests a different distribution of the pertinent characteristics, pointing toward "intergroup" distinction of older adults and their cognitive function (Smith Jr., 1970). In our Dutch sample, the older adults had a lower education level and more of a cognitive decline compared to the Swedish sample.

A further strength of this study is the relatively high mean age of the samples (initially 74 years). Many studies tend to focus on 65-75-year-olds when studying technology use. For the oldest old internet use might be more difficult to learn or handle. It is known that, with age, it can be difficult to adopt a task if it represents an unfamiliar cognitive domain. Hence, taking up the learning of a new task with computers or even guiding oneself through information seeking online can become problematic (Czaja, 2005; Park, 1992). For example, if a website is loaded with information, it could put a person off trying to learn how to navigate through the website. Or when less frequent users have gotten used to a layout in an email program or other frequented website which then changes, there is a large chance that they will not have the energy to learn to use the redesigned program or website.

It should be noted however, that old age is not the only relevant factor in both cognitive decline and technology use. Much depends on previous experience and the individual; one aging person differs from the other, and when it comes to cognitive function, there is no one-on-one relation between age and a certain cognitive score. Cognitive functioning is dependent on many factors such as genetics, environment, education, personal history, and physical and emotional capabilities. In the same vein, internet use may be beneficial for some but not for other older people. Future research needs to identify specific groups that benefit most from internet use.

For the coming generations using the internet in old age may be a given and something not even questionable. This can be positive, as this study showed that internet use was associated with less cognitive decline. For an older person, however, it could be that some aspects of what one was able to do online no longer are possible due to decline in motor skills, eyesight, or cognitive skills. Learning a new tool can be difficult in the third and fourth age, and if technology advances at the pace as it does now, it is very hard for some to keep up. If it is a question of using the same technology throughout the years, this will be an easier task. Always having to train and relearn can become tedious, and this should be considered by those developing policies, programs, and computer software.

\section{Limitations}

It should be noted that our sample only included people who answered the question on internet use at $\mathrm{T} 1$, who were 66 years of age and above, and who answered the MMSE at both time periods (T1 and T2). Those with incomplete data were older, had more functional limitations, and lived alone compared to those with complete data. It is difficult to estimate if and how this bias could have affected our findings.

Our study covers the period 2001-2010. It may be that the results are partly a cohort effect. Once the smartphone and tablet were more common in everyday use (from 2007 onwards), it might have been easier to start using the internet for some people. Thus, repeating this same study taking a sample from 2011 onwards could give different results with respect to change in cognitive function.

We used the MMSE, which is a global measure for cognitive functioning and not sensitive to subtle changes in cognitive function. There could also be a threat to external validity as the MMSE was given to the same people at least twice, possibly making them more comfortable as they were acquainted with the questions of the test. This could underestimate the change in cognitive score over time, as a person may score higher if the test would have only been given to them once, or if a different test was used at each time point.

\section{Conclusion and recommendation}

This study found an association between internet use and less cognitive decline during a period of six years (2001/2003-2007/2010). The results could be seen as a potential indication of internet use having a positive impact on healthy cognitive aging. It would, however, be useful to first replicate our findings in other studies and contexts. In particular, specific internet activities could be addressed. Also, more specific cognitive outcomes could be examined, such as information processing speed, cognitive flexibility, and magnetic resonance imaging (Slegers et al., 2009; Webster 
et al., 2017), and more pertinent confounders could be added, such as vision impairment and personality (Gell et al., 2015; Berner et al., 2017). If replicated, our findings may warrant well-designed intervention studies (Klimova, 2016; Yates et al., 2016). It has been recommended that cognition-focused interventions should implement newly acquired skills and strategies in the everyday context (Kurz et al., 2011). Moreover, multi-domain, cognitively stimulating activities have been shown to improve general cognitive functioning (Huntley et al., 2015; Ihle et al., 2017; Sherman et al., 2017; Zhu et al., 2017). Internet use, once adopted, is apt to become part of everyday activities. It involves several cognitive as well as motor functions, and many internet activities are cognitively stimulating. Thus, internet use appears to be a promising candidate for cognition-focused intervention.

\section{Conflict of interest}

None

\section{Description of authors' roles}

JB designed the study, performed the statistical analyses, and wrote drafts of the manuscript. HC, SE, A-KW, JSB, PA, and DD revised the paper critically. All the authors were involved in the final approval of the version to be published.

\section{Acknowledgments}

The Longitudinal Aging Study Amsterdam is supported by a grant from the Netherlands Ministry of Health Welfare and Sports, Directorate of LongTerm Care. For the current study, grants were received from the Lars Hiertas Foundation (Sweden), the Bavostichting Heemstede (the Netherlands), and the Stichting tot Steun VCVGZ (the Netherlands). The funders had no role in the study design; the collection, analysis, and interpretation of data; in the writing of this paper; and in the decision to submit it for publication. The authors would like to thank Najada Stringa for her help with the meta-analyses.

\section{Supplementary material}

To view supplementary material for this article, please visit https://doi.org/10.1017/S1041610219000668

\section{References}

Ashby, G., Isen, A. and Turken, U. (1999). A neuropsychological theory of positive affect and its influence on cognition. Psychological Review, 106, 529-550.
Baltes, P. B. (1997). On the incomplete architecture of human ontogeny. Selection, optimization, and compensation as foundation of developmental theory. American Psychologist, 52, 366-380.

Berner, J. et al. (2015). Factors influencing Internet usage in older adults ( 65 years and above) living in rural and urban Sweden. Health Informatics fournal, 21, 237-249. doi: $10.1177 / 1460458214521226$.

Berner, J. S. et al. (2016). A cross-national and longitudinal study on predictors in starting and stopping Internet use (2001-2013) by Swedish and Dutch older adults 66 years and above. Gerontechnology, 14, 157-168.

Berner, J., Aartsen, M. and Deeg, D. (2017). Predictors in starting and stopping Internet use between 2002 and 2012 by Dutch adults 65 years and older. Health Informatics Fournal, 19, 152-162. doi:10.1177/1460458217720398.

Cabeza, R., Anderson, N. D., Locantore, J. K. and McIntosh, AR. (2002). Aging gracefully: compensatory brain activity in high-performing older adults. Neuroimage, 17, 1394-1402.

Cohen, J. A., Verghese, J. and Zwerling, J. (2016). Cognition and gait in older people. Review. Maturitas, 93, 73-77. doi:10.1016/j.maturitas.2016.05.005.

Czaja, S. J. (2005). The impact of aging on access to technology. ACM Sigaccess Accessibility and Computing, 83, 7-11.

Ellwardt, L., Van Tilburg, T. G. and Aartsen, M. J. (2015). The mix matters: complex personal networks relate to higher cognitive functioning in old age. Social Science $\mathbb{E}$ Medicine, 125, 107-115. doi:10.1016/j.socscimed.2014.05 .007 .

Eurostat. Individuals places in internet use. Retrieved 17 September 2017 from http://ec.europa.eu/eurostat/web/ products-datasets/-/isoc_ci_ifp_pu.

Folstein, M. F., Folstein, S. E. and McHugh, P. R. (1975). "Mini-mental state". A practical method for grading the cognitive state of patients for the clinician. Fournal of Psychiatric Research, 12, 189-198.

Gell, N. M., Rosenberg, D. E., Demiris, G., LaCroix, A. Z. and Patel, K. V. (2015). Patterns of technology use among older adults with and without disabilities. Gerontologist, 55, 412-421.

Huisman, M., et al. (2011). Cohort profile: the Longitudinal Aging Study Amsterdam. International fournal of Epidemiology, 40, 868-876. doi:10.1093/ije/dyq219.

Huntley, J. D., Gould, R. L., Lui, K., Smith, M. and Howard, R. J. (2015). Do cognitive interventions improve general cognition in dementia? A meta-analysis and meta-regression. BMF Open, 5(4), e005247.

Ihle, A. et al. (2017). The relation of education, occupation, and cognitive activity to cognitive status in old age: the role of physical frailty. International Psychogeriatric, 29, 1469-1474.

Jolles, J., Verhey, F., Riedel, W. and Houx, P. (1995). Cognitive impairment in elderly people. Drugs $\&$ Aging, 7, 459-475.

Klimova, B. (2016). Use of the internet as a prevention tool against cognitive decline in normal aging. Clinical Interventions in Aging, 11, 1231-1237.

Kurz, A. F., Leucht, S. and Lautenschlager, N. T. (2011). The clinical significance of cognition-focused interventions for cognitively impaired older adults: a 
systematic review of randomized controlled trials. International Psychogeriatric, 23, 1364-1375.

Lagergren, M. et al. (2004). A longitudinal study integrating population, care and social services data. The Swedish National study on Aging and Care (SNAC). Aging Clinical and Experimental Research, 16, 158-168.

Lawton, M. P. and Brody, E. M. (1969). Assessment of older people: self-maintaining and instrumental activities of daily living. Gerontologist, 9, 179-186.

McWhinnie, J. R. (1981). Disability assessment in population surveys: results of the O.E.C.D. Common Development Effort. Revue d'Epidémiologie et de Santé Publique, 29, 413-419.

Park, D. C. (1992). Applied cognitive aging research. In: F. I. M. Craik and T. A. Salthouse (Eds.), The Handbook of Aging and Cognition (pp. 449-494). Hillsdale, NJ: Laurence Erlbaum Associates Publishers. https://psycnet .apa.org/psycinfo/1992-98157-009.

Reijnders, J., van Heugten, C. and van Boxtel, $M$. (2013). Cognitive interventions in healthy older adults and people with mild cognitive impairment: a systematic review. Ageing Research Reviews, 12, 263-275. doi:10.1016/j .arr.2012.07.003.

Rogers, E. (2003). Diffusion of Innovations. New York: Free Press.

Salthouse, T. A. (1996). The processing-speed theory of adult age differences in cognition. Psychological Review, 103, 403-428.

Sanchez-Valle, M., Vinaras-Abad, M. and LorrenteBorroso, C. (2017). Empowering the elderly and promoting active ageing through the internet: the benefit of e-inclusion programs. In Safe at Home with Assistive Technology, 6, 95-108. doi:10.1007/978-3-319-42890-1_7.

Sherman, D. S., Mauser, J., Nuno, M. and Sherzai, D. (2017). The efficacy of cognitive intervention in mild cognitive impairment (MCI): a meta-analysis of outcomes on neuropsychological measures. Neuropsychology Review, $27,440-484$.
Slegers, K., van Boxtel, M. and Jolles, J. (2009). Effects of computer training and internet usage on cognitive abilities in older adults: a randomized controlled study. Aging Clinical and Experimental Research, 21, 43-54.

Smith Jr, N. (1970). Replication studies: a neglected aspect of psychological research. American Psychologist, 25, 970-975. doi:10.1037/h0029774.

Speer, D. C. and Greenbaum, P. E. (1995). Five methods for computing significant individual client change and improvement rates: support for an individual growth curve approach. Fournal of Consulting and Clinical Psychology, 63, 1044-1048.

van Gelder, B. M., Tijhuis, M., Kalmijn, S., Giampaoli, S., Nissinen, A. and Kromhout, D. (2006). Marital status and living situation during a 5-year period are associated with a subsequent 10 -year cognitive decline in older men: the FINE Study. The fournals of Gerontology. Series B, Psychological Sciences and Social Sciences, 61, P213-P219.

Webster, L. et al. (2017). Development of a core outcome set for disease modification trials in mild to moderate dementia: a systematic review, patient and public consultation and consensus recommendations. Health Technology Assessment, 21, 1-192.

Xavier, A. J. et al. (2014). English longitudinal study of aging: can internet/E-mail use reduce cognitive decline? Fournals of Gerontology. Series A: Biological Sciences and Medical Sciences, 69, 1117-1121. doi:10.1093/gerona/ glu105.

Yates, L. A., Ziser, S., Spector, A. and Orrell, M. (2016). Cognitive leisure activities and future risk of cognitive impairment and dementia: systematic review and meta-analysis. International Psychogeriatric, 28, 1791-1806.

Zhu, X., Qiu, C., Zeng, Y. and Li, J. (2017). Leisure activities, education, and cognitive impairment in Chinese older adults: a population-based longitudinal study. International Psychogeriatric, 29, 727-739. 